\title{
Encapsulation of Iron Oxide Nanoparticles Type Core-Shell in Chitosan as Possible Theranostic Agent
}

\author{
P. Y. Sauceda-Oloño ${ }^{1}$, J. A. Lucero-Acuña ${ }^{2}$, P. Zavala-Rivera ${ }^{2}$ \\ 1. Posgrado en Nanotecnología, Departamento de Física, Universidad de Sonora (UNISON), Blvd. Luis \\ Encinas y Rosales S/N col. Centro, C.P. 83000, Hermosillo, Sonora, México \\ 2. Departamento de Ingeniería Química y Metalurgia, Universidad de Sonora (UNISON), Blvd. Luis \\ Encinas y Rosales S/N col. Centro, C.P. 83000, Hermosillo, Sonora, México
}

With the recent advances on the nanotechnology science, nanoencapsulation technique is rapidly growing. Nanocapsules are nanostructured materials composed of a core and a protective layer [1]. The core is typically solid or liquid, and the protective layer is usually a non-toxic polymer membrane, which makes the delivery of the drug controllable. Today researchers are interested in novel drug delivery systems, a feasible method is nanoencapsulation, due to its features such as: release drugs in a specific area without affecting other non-contaminated areas, preventing overdoses and leveraging the effect of the drug and its use in the diagnosis of the diseases as marker or contrast agent at the same time [1,2,3].

On the present work, we developed a protocol for the synthesis of nanoparticles (NP's) type core-shell of $\mathrm{Fe}_{3} \mathrm{C} / \gamma-\mathrm{Fe}_{2} \mathrm{O}_{3}$ encapsulated on micellar systems of sodium dodecyl sulfate (SDS) with hydrophobic core of oleic acid (OA) stabilized with chitosan (C). The NP's was synthetized using as precursor iron pentacarbonyl $\left(\mathrm{Fe}(\mathrm{CO})_{5}\right)$, and as a stabilization of the $\mathrm{OA}$ particles for their use in nanoemulsions (NE's) using sono-synthesis technique. SEM analysis were carried out with a FESEM JEOL JSM$7800 \mathrm{~F}$.

The NP's obtained after centrifugation result with hydrodynamic diameter of $19.71 \mathrm{~nm}$ and a 0.134 polydispersity index, which were used on the organic phase of the synthesis process of NE's. With a 7 $\mathrm{mM}$ concentration of surfactant SDS, we obtained NE's with hydrodynamic diameter of $223.6 \mathrm{~nm}$ and 0.216 polydispersity index. Also, NE's without NP's was synthetized resulting on NE's with hydrodynamic diameter of $229.9 \mathrm{~nm}$ and a 0.146 polydispersity index. On both experiments we obtained a zeta potential of $-41.5 \mathrm{mV}$, due the negative natural charge of the surfactant used.

After the NE's was obtained, we cover them by sonication with a dissolution of C of low molecular weight on acetic acid at 2\%. The nanocapsules with NP's have a hydrodynamic diameter of $185.3 \mathrm{~nm}$ and a 0.150 polydispersity index and the nanocapsules without NP's have a hydrodynamic diameter of $225.3 \mathrm{~nm}$ and a 0.222 polydispersity index. The zeta potential changed to $36.7 \mathrm{mV}$ and $36.2 \mathrm{mV}$, confirming the encapsulation with $\mathrm{C}$ due the positive charge of it.

The SEM and STEM analyses shows a spherical morphology (See Figures 1, 2 and 3), and the possible encapsulation of the NP's of iron oxide type core-shell. As it can be observed in Figure 3, the delimited area by the figure shows the probable encapsulation of the NP's. With the characteristics obtained of the nanocapsules, their physicochemical are good candidates for using as a treatment and diagnostic material for diseases as a theranostic agent. 


\section{References:}

[1] R. W. Kelsall, I. W. Hamley, and M. Geoghgan in "Nanoscale Science and Technology", ed. John Wiley \& Sons, (Wiley, New Jersey) p. 419.

[2] C. M. Niemeyer and C. A. Mirkin in "Nanobiotechnology: Concepts, Applications and Perspectives", ed. John Wiley \& Sons, (Wiley, New Jersey) p. 168.

[3] A. Tiwari and A. N. Nordin in "Advanced Biomaterials and Biodevices", ed. John Wiley \& Sons, (Wiley, New Jersey) p. 487.

[4] The authors acknowledge funding from CONACyT (255791-INFR-2015) to acquire the FESEM JEOL JSM-7800F microscope. P. Y. Sauceda-Oloño gratefully acknowledges the scholarship provided by CONACyT (México).

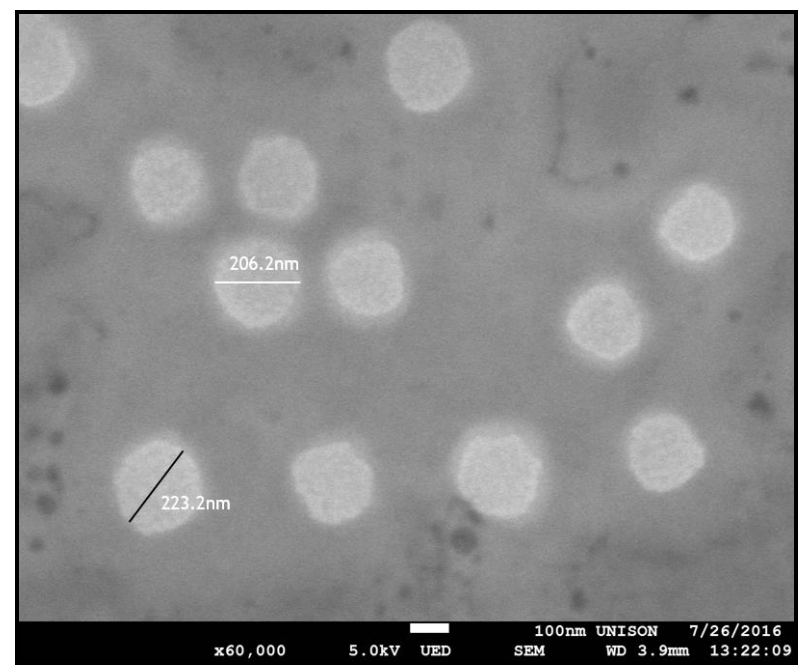

Figure 1. SEM image of nanocapsules without NP's. With a size bar of $100 \mathrm{~nm}$.

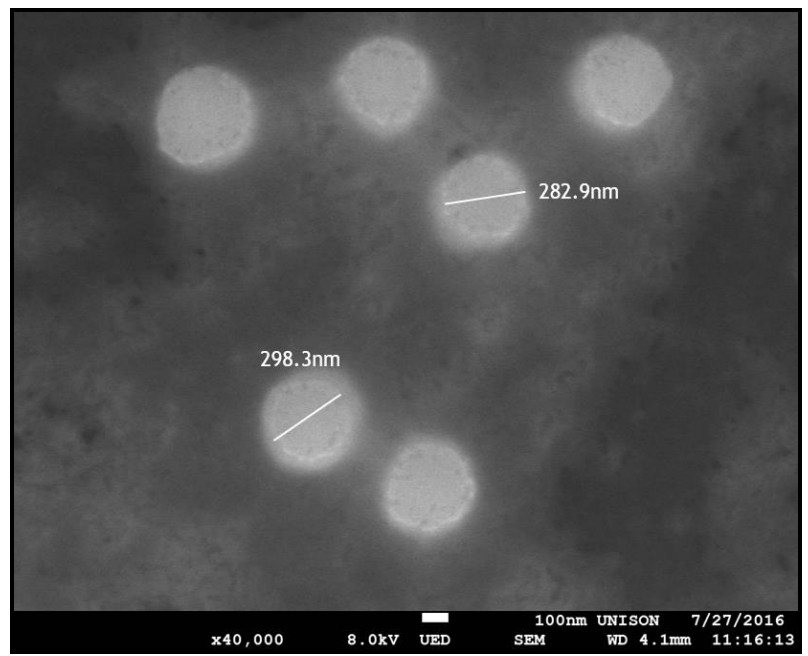

Figure 2. SEM image of nanocapsules with NP's. With a size bar of $100 \mathrm{~nm}$.

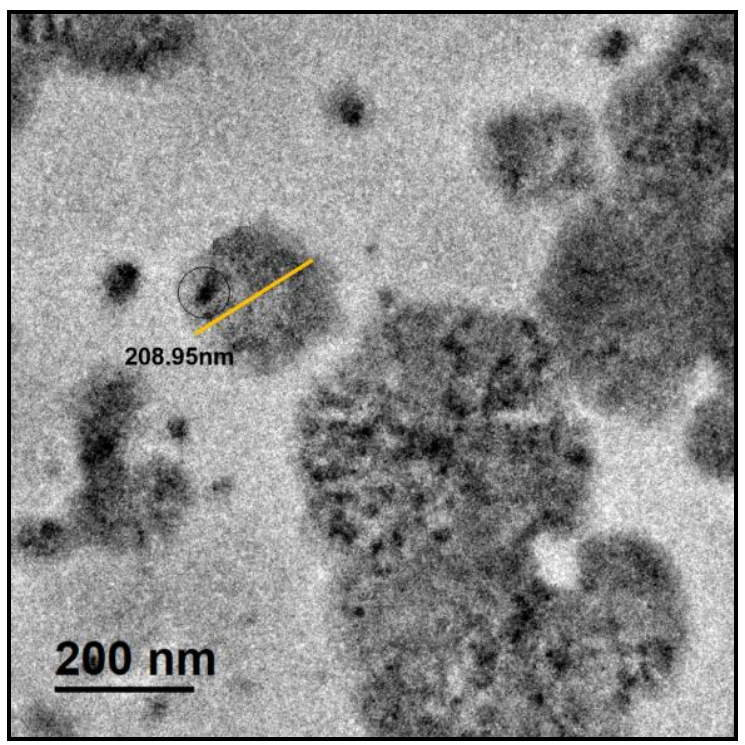

Figure 3. STEM image of nanocapsules with NP's. With a size bar of $200 \mathrm{~nm}$. 